\title{
Aktueller Stand Internalisierender Störungen im Kindes- und Jugendalter: Sind sie aus den Kinderschuhen ausgewachsen?
}

Tina In-Albon

Klinische Psychologie und Psychotherapie, Universität Basel, Schweiz

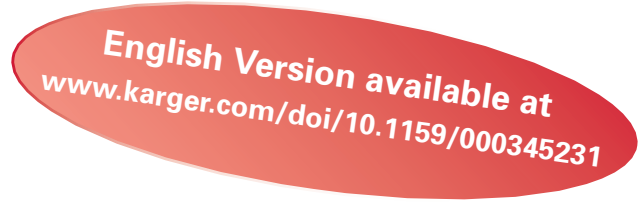

\section{Schlüsselwörter}

Internalisierende Störungen - Angststörungen im Kindes- und Jugendalter . Depression im Kindesund Jugendalter · Psychotherapie - Prävention . Psychotherapieforschung $\cdot$ Klassifikation

\section{Zusammenfassung}

Internalisierende Störungen sind die häufigsten psychischen Störungen im Kindes- und Jugendalter. Es kann heute als erwiesen angesehen werden, dass Angst- und depressive Störungen nicht auswachsen, sondern im Gegenteil stabil sind und einen beträchtlichen Risikofaktor für die Entwicklung weiterer psychischer Störungen im Erwachsenenalter darstellen. Daher sollte diesen Störungen in der Früherkennung, Prävention und Versorgung vermehrt Beachtung geschenkt werden, da diese Kinder häufig zu lange im Stillen leiden, bevor sie adäquate Hilfe erhalten. Für eine Verbesserung der Versorgungslage sollte eine Reihe von Fachpersonen mit einbezogen werden, die mit Kindern und Jugendlichen in regelmäßigem Kontakt stehen. Zudem sollten auch Kinder und Jugendliche selbst über psychische Probleme aufgeklärt werden. Sind Angst- und depressive Störungen erkannt, liegen gut überprüfte Therapien vor, die auch nachhaltig wirksam sind. Nebst der Verbesserung der Früherkennung und Steigerung der Wirksamkeit von Interventionsprogrammen sollten Präventionsprogramme zur Verbesserung der allgemeinen psychischen Gesundheit gefördert und durchgeführt werden.

\section{Keywords}

Internalizing disorders . Anxiety disorders in children and adolescents - Depression in children and adolescents - Psychotherapy - Prevention . Psychotherapy research $\cdot$ Classification

\section{Summary}

State of Research on Internalizing Disorders in Children and Adolescents: Is It Still in Its Infancy?

Internalizing disorders are the most common mental disorders in children and adolescents. It is a fact that anxiety disorders and depression do not vanish over time. On the contrary, they are stable and represent significant risk factors for the development of further mental disorders in adulthood. Therefore, these disorders deserve more attention regarding early recognition, prevention and care, as these children often suffer in silence for too long before receiving adequate professional help. To improve their supply situation, various specialists who are in regular contact with children and adolescents should be included in the process. Furthermore, children and adolescents themselves should be educated about mental problems. If anxiety and depressive disorders are recognized, several well-evaluated treatment programs are available, which have demonstrated sustained effects. In addition, improvements in early recognition and enhancements in efficacy and effectiveness of treatment and prevention programs should be supported and warranted.

\section{KARGER \\ Fax +497614520714 \\ Information@Karger.de}

www.karger.com (c) 2012 S. Karger GmbH, Freiburs

$1016-6262 / 12 / 0224-0246 \$ 38.00 / 0$

Accessible online at:

www.karger.com/ver 


\section{Einleitung}

Im Editorial für das Themenheft «Verhaltenstherapie bei Kindern und Jugendlichen» der Zeitschrift VerhaltenstheRAPIE [Schneider, 2000] im Jahre 2000 stellte Silvia Schneider die Frage: «Steckt die Verhaltenstherapie bei Kindern noch in den Kinderschuhen?» Die Antwort auf diese Frage kann mit «ja» vorweggenommen werden. Die 4 aufgeführten Gründe für die damalige Vernachlässigung psychischer Störungen im Kindes- und Jugendalter lauteten: 1) Psychische Störungen wachsen sich aus und erfordern daher nicht speziell Beachtung, 2) die geringe Versorgungslage von Kindern mit psychischen Störungen, 3) Schwierigkeiten in der Forschung mit Kindern (zu aufwendig und damit mit einigen ethischen Problemen einhergehend) und 4) in der Psychotherapieforschung stellen kontrollierte Gruppenvergleiche die Ausnahme und nicht die Regel für den Wirksamkeitsnachweis dar.

Zwölf Jahre später möchte ich anhand aktueller Literatur auf einige der aufgeführten Gründe eingehen und die Frage umformulieren: «Ist die Verhaltenstherapie bei Kindern aus den Kinderschuhen ausgewachsen?» Zusätzlich zur Schilderung des aktuellen Standes aus den Bereichen Epidemiologie und Psychotherapieforschung werde ich auf die Klassifikation, Prävention und Therapieinhalte eingehen. Dabei werde ich mich in diesem Überblick auf Angst- und depressive Störungen beschränken.

\section{Epidemiologischer Überblick}

Die Mehrheit der Übersichtsarbeiten zur Epidemiologie psychischer Störungen im Kindes- und Jugendalter fand Lebenszeitprävalenzraten von ca. $20 \%$, sodass eine relative Einheitlichkeit dieser Prävalenzrate als gültig angenommen werden kann, trotz methodischer Unterschiede über die Studien hinweg [z.B. Ihle und Esser, 2002; Petermann, 2005; Steinhausen et al., 1998]. Diese Prävalenzrate wird auch durch eine aktuelle Studie aus den USA mit 10123 Jugendlichen zwischen 13 und 18 Jahren belegt, aus der eine Prävalenzrate von 22,2\% für psychische Störungen resultiert [Merikangas et al., 2010]. Weniger Studien liegen zu Prävalenzraten bei jüngeren Kindern vor, jedoch zeigen sich auch da bereits nachdenklich stimmende Häufigkeiten. In einer Studie von Carter et al. [2010] zeigte sich bei 6-Jährigen, dass jedes 5. Kind die Kriterien für eine psychische Störung erfüllte $(21,6 \%)$, die mit einer Beeinträchtigung einherging. Ohne Beeinträchtigung ergab sich eine Häufigkeit von $32 \%$. Kuschel et al. [2008] untersuchten 3- bis 6-jährige Kindergartenkinder in Deutschland. Je nach Beurteiler (Eltern, Erzieher) lagen die Prävalenzraten zwischen 0,5 und 9,4\%, wobei internalisierende Störungen deutlich häufiger angegeben wurden als externalisierende. Diese Verteilung zeigt sich konsistent in epidemiologischen Studien, anhand derer deutlich wird, dass im Kindes- und Jugendalter Angststörungen die häufigsten psychischen Störungen sind, gefolgt von Aufmerksamkeits- defizit- und Hyperaktivitätsstörungen (ADHS) sowie aggressiven Verhaltensstörungen. Angst- und depressive Störungen werden zu internalisierenden Störungen zusammengefasst, aggressive Verhaltensstörungen und ADHS zu externalisierenden Störungen. In einer mit Jugendlichen und jungen Erwachsenen durchgeführten Studie [Wittchen et al., 1998] wurden affektive Störungen und Angststörungen etwa gleich häufig diagnostiziert. Während bei Kindern unter 13 Jahren weniger als 3\% von Depressionen betroffen sind, steigen die Prävalenzraten bei Jugendlichen im Alter zwischen 13 und 18 Jahren auf $6 \%$ an [Costello et al., 2006]. Hinsichtlich Geschlechterunterschieden zeigen epidemiologische Studien, dass im Kindesalter internalisierende Störungen bei Mädchen und Jungen gleich häufig auftreten, dass aber ab dem Jugendalter weibliche Jugendliche höhere Prävalenzraten internalisierender Störungen aufweisen als männliche Jugendliche [Merikangas et al., 2010; Steinhausen et al., 1998]. Bezüglich des ersten Auftretens psychischer Störungen zeigen Studien, dass die Mehrheit von Erwachsenen mit psychischen Störungen bereits im Jugendalter die Diagnose einer psychischen Störung hatte. In der prospektiven Studie von Kim-Cohen et al. [2003] zeigte sich, dass 50\% der 26-Jährigen mit der aktuellen Diagnose einer psychischen Störung bereits im Alter zwischen 11 und 15 Jahren eine psychische Störung hatten. Dabei treten Angststörungen deutlich früher auf als affektive Störungen [Kessler et al., 2005; Wittchen et al., 2000a]. Im Folgenden wird auf die in der Einleitung aufgeführten 4 Gründe für die Vernachlässigung psychischer Störungen im Kindes- und Jugendalter Bezug genommen.

\section{Wachsen sich psychische Störungen im Kindes- und Jugendalter von alleine aus?}

Der erste von Schneider [2000] aufgeführte Grund für die Vernachlässigung psychischer Störungen bei Kindern und Jugendlichen war die lange Zeit vorherrschende Meinung, dass sich Verhaltensprobleme «von alleine wieder auswachsen». Seit Erscheinen des Editorials sind mehrere retro- und prospektive Studien erschienen, die eindrücklich nachweisen konnten, dass psychische Störungen im Kindes- und Jugendalter, insbesondere Angststörungen, einen beträchtlichen Risikofaktor für die Entwicklung psychischer Störungen im Erwachsenenalter darstellen [In-Albon, 2011]. Woodward und Fergusson [2001] zeigten, dass Jugendliche mit einer Angststörung ein erhöhtes Risiko hatten, als junge Erwachsene Angst- oder affektive Störungen zu entwickeln, drogenabhängig zu werden und schwache schulische Leistungen zu erbringen. Dabei ist das Spektrum der sich infolge von Angststörungen entwickelnden Störungen sehr breit: diese reichen von Angst- und depressiven Störungen über Schmerzstörungen bis zu Alkoholabhängigkeit [z.B. Brückl et al., 2007; Copeland et al., 2009]. Ergebnisse zum Verlauf von Angststörungen gehen einher mit der Stabilität. In der Übersichtsarbeit von Ihle und Esser [2002] wiesen psychische Störungen über 
alle Altersstufen eine hohe Stabilität auf. Die Stabilitätsrate emotionaler Störungen zeigte, dass ungefähr die Hälfte psychisch auffälliger 13-Jähriger auch noch im Alter von 18 Jahren emotionale Störungen aufwies. Es liegen jedoch auch kontroverse Befunde vor. In der Early Developmental Stages of Psychopathology (EDSP)-Studie mit 14- bis 24-Jährigen erfüllten zum 2-Jahres-Follow-Up nur noch 19,7\% der Betroffenen zur Baseline-Erhebung die Kriterien für eine Angststörung [Wittchen et al., 2000b], wobei sich eine große Varianz zwischen den unterschiedlichen Angststörungen zeigte. Die Frage, ob es diesen Jugendlichen im späteren Verlauf gut geht, muss jedoch verneint werden. Nur gerade $10 \%$ der Jugendlichen, die zur Baseline-Erhebung die Diagnose einer Spezifischen Phobie erfüllten, hatten 10 Jahre danach keine Störung mehr. 41\% der Jugendlichen berichtete von Spezifischen Phobien, und bei insgesamt $73 \%$ wurde eine andere Angst- oder depressive Störung diagnostiziert [Emmelkamp et al., 2009]. Ähnlich verhält sich der Verlauf bei depressiven Störungen. Unbehandelt ist der Verlauf der Depression meist chronisch rezidivierend, sodass nach einer gewissen Verbesserung zwischen 40 und $90 \%$ der betroffenen Kinder und Jugendlichen mindestens eine weitere depressive Episode erleben [Birmaher et al., 1996].

Es kann somit festgehalten werden, dass sich psychische Störungen im Kindes- und Jugendalter nicht von alleine auswachsen, sondern im Gegenteil die weitere Entwicklung stark beeinflussen.

\section{Die Versorgungslage}

Eine frühzeitige Erkennung ist eine zentrale Voraussetzung für eine optimale Versorgung betroffener Kinder und Jugendlichen. Dabei sind gerade die internalisierenden Störungen schwierig von außen zu erkennen, da sich ihre Kernsymptome auf Beeinträchtigungen des inneren Erlebens und vermeidendes Verhalten beziehen. Einer deutschen [Essau, 2005] und einer amerikanischen Studie [National Institute of Mental Health (NIMH), 2001] zufolge erhalten nur etwa 20\% aller Kinder und Jugendlichen, die wegen psychischer Probleme als behandlungsbedürftig eingestuft wurden, tatsächlich professionelle Hilfe. Des Weiteren konnte in einer deutschen Studie [Meltzer et al., 2000] nachgewiesen werden, dass Kinder mit externalisierenden Störungen häufiger professionelle Hilfe erhalten als Kinder mit internalisierenden Problemen. Diese Überlegungen gehen einher mit der Frage, wie psychische Störungen im Kindes- und Jugendalter besser und früher erkannt werden können. Dabei kommt Pädiatern eine wichtige, aber auch schwierige Rolle zu. Pädiater sind meistens die erste Anlaufstelle für Kinder und Jugendliche mit psychischen Störungen oder deren Eltern, da sich psychische Störungen häufig durch körperliche Beschwerden zeigen können. Die Ergebnisse einer Umfrage bei Pädiatern in der Schweiz [In-Albon et al., 2010] zeigten, dass das Vorhandensein von psychischen Störungen in der pädiatrischen Praxis auf 15\%, das Vorkommen internalisierender Störungen auf 7\% und das externalisierender Störungen auf $9 \%$ geschätzt wurde. Zudem zeigte sich, dass ADHS sehr häufig in der pädiatrischen Praxis diagnostiziert wird und sich die Pädiater bei der Diagnostik dieser Störung sicher fühlen. Im Vergleich zu externalisierenden Störungen waren internalisierende Störungen ebenfalls häufig, jedoch fühlten sich die Pädiater bei Diagnosen von Angst- und depressiven Störungen unsicher und wünschten sich diesbezüglich Weiterbildungen.

Zusammenfassend muss im Hinblick auf die Erkennung und Versorgung, wie bereits im Jahr 2000, noch heute von einer deutlichen Unterversorgung und Nicht-Erkennung von internalisierenden Störungen bei Kindern und Jugendlichen ausgegangen werden. Dies trifft zu, obwohl das Weiterbildungsangebot für Kinder- und Jugendlichenpsychotherapeuten mit Schwerpunkt Verhaltenstherapie in den letzten Jahren stetig ausgebaut wurde und Psychotherapieambulanzen eröffnet wurden. Ein Grund für diesen Missstand, spezifisch für die Schweiz, ist, dass die psychologische Psychotherapie nicht über die Grundversicherung abgedeckt ist, und somit eine Psychotherapie bei einem Psychologen mit erheblichen Kosten einhergehen kann. Immerhin wird per Januar 2013 der Titelschutz für Psychologen und Psychotherapeuten eingeführt. Da die Versorgung in Deutschland jedoch nicht besser ist, gibt es noch weitere Hindernisse, eine psychologische Behandlung in Anspruch zu nehmen oder nehmen zu können, die zukünftig erforscht werden müssen. Da auch bei einer guten Versorgung mit Psychotherapie noch nicht davon ausgegangen werden kann, dass die durchgeführten Therapien evidenzbasiert sind, ist die Verbreitung evidenzbasierter Therapien eine zentrale Aufgabe. Eine Vorreiterrolle für die Verbesserung des Zugangs zu evidenzbasierten Methoden nimmt derzeit England ein. Nach ersten Erfahrungen mit dem Erwachsenenprogramm gibt es nun auch einen Fokus auf Kinder und Jugendliche. Ein Hauptmerkmal des Projekts ist die Ausbildung von Fachpersonen in evidenzbasierten Methoden (aktuell für die Bereiche Elternschulungen bei oppositionellem Verhalten, Verhaltenstherapie bei Angststörungen und Depressionen im Kindes- und Jugendalter). Zusätzlich wird regelmäßige Supervision angeboten. Im Hinblick auf die Nachhaltigkeit ist ein zentraler Punkt des gesamten Projekts, dass vorgegebene Therapieerfolgsmaße während und nach der Therapie eingeholt und eingereicht werden müssen. Die ersten Ergebnisse im Bereich der Kinder- und Jugendlichenpsychotherapie können mit Spannung erwartet werden (www.iapt.nhs.uk).

\section{Psychotherapieforschung}

Seit dem Erscheinen des Themenhefts im Jahr 2000 zur Verhaltenstherapie im Kindes- und Jugendalter hat die Anzahl an Wirksamkeitsnachweisen mit kontrollierten Gruppenverglei- 
Tab. 1. Meta-Analysen und ihre Effektstärken zur Wirksamkeit von Psychotherapie bei Angst- und depressiven Störungen
Effektstärke nach Cohen's d ${ }^{\text {a }}$

\begin{tabular}{|c|c|}
\hline \multicolumn{2}{|l|}{ Angststörungen } \\
\hline In-Albon und Schneider [2007] & $\mathrm{d}=0,86(\mathrm{n}=24)^{\mathrm{b}}, \mathrm{FU} \mathrm{d}=1,36$ \\
\hline Silverman et al. [2008] & $\mathrm{d}=0,91(\mathrm{n}=4)$ \\
\hline \multicolumn{2}{|l|}{ Depressive Störungen } \\
\hline Reinecke et al. [1998] & $\mathrm{d}=1,02(\mathrm{n}=6), \mathrm{FU} \mathrm{d}=0,61$ \\
\hline Lewinsohn und Clarke [1999] & $\mathrm{d}=1,27(\mathrm{n}=12)$ \\
\hline Weisz et al. [2006] & $\begin{array}{l}\mathrm{d}=0,34(\mathrm{n}=35 \text {, inklusive Präventionsstudien und unterschiedlichem } \\
\text { Schweregrad der Depression) }\end{array}$ \\
\hline Klein et al. [2007] & $\mathrm{d}=0,53(\mathrm{n}=11)$ \\
\hline
\end{tabular}

chen stark zugenommen, sodass im Gegensatz zum Jahr 2000 randomisierte kontrollierte Therapiestudien (RCTs) auch in der klinischen Kinder- und Jugendlichenpsychotherapie die Regel und nicht mehr die Ausnahme darstellen. Aufgrund der großen Anzahl an Studien liegen bereits mehrere Übersichtsarbeiten vor, die die Wirksamkeit von Psychotherapie bei Angststörungen [In-Albon und Schneider, 2007; Silverman et al., 2008] und depressiven Störungen [Reinecke et al., 1998; Weisz et al., 2006] bei Kindern und Jugendlichen untersucht haben. Tabelle 1 gibt einen Überblick über die Effektstärken von Meta-Analysen zur Wirksamkeit von Psychotherapie bei Angst- und depressiven Störungen. Es kann somit festgehalten werden, dass die Psychotherapie bei Angststörungen im Kindes- und Jugendalter und bei depressiven Störungen im Jugendalter wirksam ist [z.B. James et al., 2005], wobei bei den Angststörungen nur die Kognitive Verhaltenstherapie (KVT) die Kriterien als evidenzbasiertes Psychotherapieverfahren erfüllt. Bezüglich der Wirksamkeit psychoanalytischer Therapie oder systemischer Therapie liegen bislang kaum Hinweise vor [American Academy of Child and Adolescent Psychiatry (AACAP), 2007]. Der Wissenschaftliche Beirat Psychotherapie hat zwar 2008 die Systemische Therapie für den Bereich Affektive Störungen und Belastungsstörungen bei Kindern und Jugendlichen anerkannt, doch beruhte diese Bewertung fast ausschließlich auf Studien zu Depressionen des Kindes- und Jugendalters und nicht zu Angststörungen. Eine Therapiestudie zu Angststörungen des Kindes- und Jugendalters zeigte, dass die Kombination von KVT und Systemischer Therapie im Vergleich zur «puren» KVT keine besseren Therapieerfolge verzeichnen konnte [Siqueland et al., 2005]. Gemäß des Berichts des Wissenschaftlichen Beirats liegen für Gesprächspsychotherapie, Psychodrama, Neuropsychologie, Eye Movement Desensitization and Reprocessing (EMDR) und Hypnotherapie für den Bereich Kinder und Jugendliche derzeit keine hinreichenden Wirksamkeitsnachweise vor; sie sind somit nicht als Behandlungsmethode bei Angststörungen des Kindes- und Jugendalters indiziert (www.wbpsychotherapie.de). Zusätzlich zur Wirksamkeit kann auch die Nachhaltigkeit der Therapie- effekte bei Angststörungen als gegeben angesehen werden [Kendall et al., 2004; Saavedra et al., 2010]. Zudem beschränken sich die Behandlungserfolge nicht nur auf die Angstsymptomatik, sondern äußern sich auch in der Reduktion der depressiven Symptomatik [In-Albon und Schneider, 2007]. Im Bereich der Angststörungen ist einschränkend festzuhalten, dass die in den Meta-Analysen zusammengefassten Studien die «efficacy-Wirksamkeit» (Wirksamkeit unter Laborbedingungen) und nicht die «effectiveness-Wirksamkeit» (Alltagswirksamkeit) untersucht haben. Des Weiteren wurden in die Therapiestudien vorwiegend Kinder im Schulalter einbezogen, sodass die Frage offen bleibt, inwiefern die Verhaltenstherapie auch bei Kindern im Vorschulalter wirksam ist. Ein weiterer Punkt ist, dass die meisten Therapiestudien Kinder mit unterschiedlichen Angststörungen (Störung mit Trennungsangst, Soziale Phobie, Generalisierte Angststörung) zusammengefasst haben und es im Vergleich zu den Erwachsenen wenig störungsspezifische RCTs im Bereich der Angststörungen gibt. Auf diese Punkte wird im Folgenden eingegangen. Die effectiveness-Forschung steht noch immer in ihren Anfängen, obwohl bereits 1999 eine Therapiestudie publiziert wurde, in der eine Effektstärke von -0,08 für traditionelle Therapien gemessen wurde [Weiss et al., 1999]. Dieser Effekt verbesserte sich in einer Katamneseuntersuchung nach 2 Jahren nur geringfügig [Weiss et al., 2000]. Bachmann et al. [2010] untersuchten die Alltagswirksamkeit im naturalistischen ambulanten Setting in 9 Kinder- und Jugendpsychiatrischen Praxen. Kleine bis moderate Therapieeffekte wurden für die Angststörungen und ADHS berichtet, keine signifikanten Effekte für depressive Störungen und die Störung des Sozialverhaltens. Kinder mit Angststörungen im Vorschulalter wurden in kürzlich erschienenen Therapiestudien untersucht [Hirshfeld-Becker et al., 2010; Schneider et al., 2011]. In einer amerikanischen Therapiestudie mit einem Wartelistekontrolldesign für Kinder zwischen 4 und 7 Jahren wurden unterschiedliche Angststörungen (Störung mit Trennungsangst, Soziale Phobie, Agoraphobie, Spezifische Phobie) zusammengefasst. Die Responder-Rate der Intent-to-TreatAnalyse lag bei 59\% [Hirshfeld-Becker et al., 2010]. Die Stu- 
die von Schneider et al. [2011] ist eine randomisierte und kontrollierte Psychotherapiestudie mit Kindern zwischen 5 und 7 Jahren, die an einer Störung mit Trennungsangst leiden. Die 43 Kinder mit einer primären Diagnose einer Störung mit Trennungsangst wurden entweder einer 16-wöchigen störungsspezifischen Therapie oder einer 12-wöchigen Warteliste zugeteilt. Die Ergebnisse zeigten, dass $76 \%$ der Kinder nach der Behandlung die Diagnosekriterien für die Störung mit Trennungsangst nicht mehr erfüllten und das Vermeidungsverhalten in Trennungssituationen aus Sicht der Kinder, Mütter und Väter signifikant abnahm.

Für eine verbesserte Wirksamkeit von Therapien sollten zukünftig weitere Verfahren entwickelt und evaluiert werden, wie beispielsweise die computerunterstützte Verhaltenstherapie [z.B. Khanna und Kendall, 2010; Spence et al., 2011] oder die Modifikation von kognitiven Verzerrungen [Cowart und Ollendick, 2011; In-Albon und Schneider, 2012a].

\section{Störungstypische Psychotherapiestudien}

In den Kinderschuhen steckt im Kinderbereich der Angststörungen noch immer die Störungsspezifität. Die meisten Therapiestudien fassen Kinder mit unterschiedlichen Angststörungen zusammen, sodass wenige störungsspezifische Kenntnisse im Grundlagenbereich, aber auch in der Therapieforschung zu den einzelnen Angststörungen, vorliegen. Eine Ausnahme stellt die Soziale Phobie dar. Für die Soziale Phobie haben Kremberg und Mitte [2005] einen Überblick zur Wirksamkeit der KVT erstellt. Im Prä-Post-Vergleich der Behandlungs- und Kontrollgruppe zeigte sich über 9 Studien hinweg eine hohe Effektstärke (Hedges $g=0,82$ ). 2 aktuelle Therapiestudien aus Deutschland für Kinder mit Sozialer Phobie berichteten vielversprechende Daten zur Wirksamkeit und Stabilität der Ergebnisse [Tuschen-Caffier et al., 2010; Melfsen et al., 2011]. Für die Spezifische Phobie liegt eine amerikanisch-schwedische 1-Session-Therapiestudie vor [O1lendick et al., 2009]. Es wurden 2 aktive Therapiebedingungen (1-Session-Behandlung vs. Aufmerksamkeit) und 1 Wartelistekontrollgruppe miteinander verglichen. Die 1-SessionBehandlung dauerte maximal $3 \mathrm{~h}$ und beinhaltete die Bearbeitung katastrophisierender Gedanken, graduierte Konfrontation in vivo und Verhaltenstests. Die Aufmerksamkeitsbedingung dauerte gleich lange, beinhaltete aber keine In-vivo-Konfrontationen. Die Effekte der 1-Session-Behandlung $(\mathrm{d}=1,84)$, aber auch jene der Aufmerksamkeitsbedingung $(\mathrm{d}=1,23)$ können als hoch interpretiert werden. Gute Effekte $(\mathrm{d}=0,58)$ der Therapiekomponente Psychoedukation zeigten sich auch in unserer Meta-Analyse [In-Albon und Schneider, 2007].

In der Therapieforschung von Angst- und depressiven Störungen sind 2 große Therapiestudien zu nennen, die die Wirksamkeit von Psychotherapie, Psychopharmakotherapie und der kombinierten Behandlung untersucht haben: die «Treat- ment for Adolescents with Depression Study» (TADS) [Treatment for Adolescents with Depression Study (TADS) Team, 2007] zur Behandlung von Depressionen und die «Child-Adolescent Anxiety Multimodal Study» (CAMS) [Walkup et al., 2008] für die Behandlung von Angststörungen. Die Gemeinsamkeiten der Studien sind der multizentrische Ansatz und das Studiendesign mit 4 Bedingungen (KVT alleine, Psychopharmaka (Serotonin-Wiederaufnahmehemmer (SSRI)) alleine, KVT und SSRI kombiniert, Placebo-Bedingung). Zusätzlich wird die «Treatment of SSRI-Resistant Depression in Adolescents» (TORDIA)-Studie [Brent et al., 2008] beschrieben, die mit depressiven Jugendlichen durchgeführt wurde, die nicht auf die primäre Behandlung mit SSRIs angesprochen haben.

In der CAMS bestand die Stichprobe aus 488 Kindern im Alter zwischen 7 und 17 Jahren mit einer primären Angstdiagnose (Störung mit Trennungsangst, Generalisierte Angststörung oder Soziale Phobie). Ausgeschlossen wurden Kinder mit Schulvermeidung aufgrund von Angst und einem früheren Nichtansprechen auf eine SSRI- oder eine KVT-Behandlung. Die Kinder wurden randomisiert den 4 Bedingungen zugeordnet. Die KVT-Bedingung bestand aus 14 Sitzungen (12 Sitzungen mit dem Kind/Jugendlichen, 2 Sitzungen mit den Eltern, Dauer 12 Wochen) unter Anwendung des Manuals «Coping Cat» [Kendall und Hedtke, 2006], der medikamentösen Behandlung (12 Wochen SSRI Sertralin, Dosis-Range 25200 mg/Tag, 8 Sitzungen), der kombinierten KVT- und SSRIBedingung und der Placebo-Bedingung. Haupterfolgsmaß der Studie war die allgemeine globale Verbesserungsskala, die vom Therapeuten ausgefüllt wurde. Dabei zeigte sich unter der Kombinationsbedingung eine Verbesserung von 80,7\%, unter der KVT-Bedingung 59,7\%, unter der Sertralin-Bedingung 54,9\% und unter der Placebo-Bedingung 23,7\%. Somit zeigte sich eine Überlegenheit der Kombinationsbedingung. Bei genauerer Betrachtung der Ergebnisse zeigte sich unter der KVT-Bedingung, dass diese Kinder nach der Therapie weniger Schlafstörungen, Müdigkeit und Ruhelosigkeit aufwiesen sowie weniger Therapieabbrüche auftraten. Kritisch anzumerken ist, dass das Coping-Cat-Programm mit 16 Sitzungen seine Wirksamkeit nachweisen konnte und nicht mit 14 Sitzungen [Kendall, 1994; Kendall et al., 1997]. Weniger Therapiesitzungen könnte eine mögliche Erklärung für die relativ niedrige Effektstärke der KVT sein (Hedges g = 0,31). Bei der Wirksamkeit der SSRI-Behandlung gibt es Hinweise, dass Jugendliche stärker profitieren als Kinder [Bridge et al., 2007]. Jedoch ist die Wirksamkeit bei Jugendlichen im Vergleich zu Erwachsenen deutlich niedriger [Steinhausen, 2010]. Für Schlussfolgerungen und Handlungsempfehlungen sollten die Katamnesedaten der Studie abgewartet werden. Für einen Überblick zur Pharmakotherapie bei Kindern und Jugendlichen mit Angststörungen siehe Rynn et al. [2011].

Die TADS-Stichprobe bestand aus 439 Kindern im Alter zwischen 12 und 17 Jahren mit einer primären Diagnose einer Major Depression. Direkt nach der Therapie [TADS, 2004] 
zeigte sich eine Überlegenheit der Kombinationsbehandlung (KVT und SSRI) und der medikamentösen Behandlung mit Fluoxetin. Die KVT war weniger effektiv als diese beiden Bedingungen und nicht signifikant wirksamer als die PlaceboBedingung. Betrachtet man jedoch die Katamnesedaten nach 36 Wochen, sieht das Bild differenzierter aus. Die ResponderRaten lagen nach 36 Wochen für die Kombinationsbehandlung bei $86 \%$, bei $81 \%$ für die SSRI-Behandlung und bei $81 \%$ für die KVT, d.h. im Langzeitverlauf zeigten sich keine signifikanten Unterschiede mehr zwischen den Behandlungen. Die Kombinationsbehandlung bzw. die SSRI-Behandlung beschleunigten das Ansprechen auf die Therapie, während der Zusatz KVT zur Medikation deren Sicherheit erhöhte. Brent [2006] merkte kritisch an, dass die Effektstärke der KVTBehandlung, verglichen mit anderen Effektstärken von Therapiestudien bei Depressionen, geringer ausfiel, und dass viele Therapiekomponenten in jeweils geringer Dosis nicht unbedingt $\mathrm{zu}$ einer besseren Wirksamkeit führen, entsprechend dem Sprichwort «weniger ist oft mehr».

TORDIA [Brent et al., 2008] fokussierte auf Jugendliche mit depressiven Störungen, die nicht auf die primäre Behandlung mit SSRIs angesprochen haben. Die Jugendlichen wurden zufällig 4 Bedingungen zugeteilt: 1) Wechsel zu einem anderen SSRI, 2) Wechsel zu einem anderen SSRI plus KVT, 3) Wechsel zu Venlafaxin, 4) Wechsel zu Venlafaxin plus KVT. 334 Jugendliche nahmen an der Studie teil, ihr mittleres Alter betrug 16 Jahre (12-18 Jahre). Die Ergebnisse zeigten, dass sich für Jugendliche, die in einer ersten Behandlung nicht auf ein SSRI ansprachen, der Wechsel zu einem anderen Antidepressivum in Kombination mit KVT am wirksamsten erwies [Brent et al., 2008].

Bezüglich der Wirksamkeit von Psychotherapie bei Jugendlichen mit leichten und mittelschweren Depressionen kann die KVT und die Interpersonelle Therapie (IPT) empfohlen werden [Ihle et al., 2004]. In 2 Studien zeigten sich positive Effekte der IPT [Mufson et al., 1999; Rosselló und Bernal, 1999] und der Familientherapie [Brent et al., 1997; Diamond et al., 2002]. Langfristig zeigten sich hingegen weniger positive Ergebnisse in der Wirksamkeit psychotherapeutischer Behandlungsverfahren [Pössel und Hautzinger, 2006]. Die Evidenzbasierung der Depression im Kindesalter ist als unbefriedigend einzustufen [Ihle et al., 2004]. Gegenwärtig liegen keine Psychotherapiestudien aus dem deutschsprachigen Raum vor, sodass die vorliegenden Behandlungsmanuale, die auf Deutsch erhältlich sind [Ihle und Herrle, 2003; Harrington, 2001], bezüglich ihrer Komponenten empfohlen werden können, aber ihre Effektivität, insbesondere auch die längerfristige Wirksamkeit, noch nachweisen müssen.

\section{Setting in der Psychotherapie}

In der Psychotherapie mit Kindern und Jugendlichen ist die Frage nach dem Setting, in dem die Therapie durchgeführt wird, eine wichtige Komponente. In der Angst- und Depressionsbehandlung zeigen sich keine Unterschiede bezüglich der Wirksamkeit, ob die Therapie individuell oder in der Gruppe durchgeführt wird [In-Albon und Schneider, 2007; Silverman et al., 2008; Pössel und Hautzinger, 2006]. Eine zusätzliche Setting-Variable stellt, im Unterschied zur Psychotherapie mit Erwachsenen, die Frage des Einbezugs der Eltern in die Angstbehandlung der Kinder dar. Aufgrund der Forschungsbefunde, dass verschiedene Elternfaktoren wie ein kritisches Elternverhalten, Angststörungen der Eltern oder eine konfliktreiche Eltern-Kind-Beziehung bei der Entstehung und Aufrechterhaltung von Angststörungen eine Rolle spielen [Hudson und Rapee, 2005; Schneider et al., 2009], könnte man davon ausgehen, dass Eltern in die Behandlung ihrer Kinder miteinbezogen werden sollten. Mehrere Übersichtsarbeiten konnten jedoch keine unterschiedliche Wirksamkeit zwischen Therapien zeigen, die kindfokussiert oder mit Einbezug der Eltern durchgeführt wurden [Casey und Berman, 1985; In-Albon und Schneider, 2007; Silverman et al., 2008]. Interessanterweise zeigte sich in der Studie von Silverman et al. [2009], dass sich auch unter der kindfokussierten Bedingung die mütterliche Angst reduzierte sowie sich die ElternKind-Beziehung verbesserte. Die Autoren gehen von einem reziproken Einfluss zwischen Kind- und Elternvariablen aus. Für die Praxis stellt sich daher die Frage: Für welches Kind bzw. welche Familie ist welches Setting am wirksamsten? Gegenwärtig können folgende Empfehlungen abgegeben werden: Die Eltern von jüngeren Kindern und Eltern, die selbst ängstlich sind, sollten in die Therapie der Kinder miteinbezogen werden [Bodden et al., 2008]. Im Hinblick auf die Ängstlichkeit der Eltern braucht es jedoch weitere Forschung zur Frage, wann die Eltern eine Therapie für sich selbst in Anspruch nehmen sollten. Für den kindfokussierten Ansatz spricht die klinische Erfahrung, dass für eine erfolgreiche Therapie die Steigerung des Selbstwerts und der Selbstwirksamkeitsüberzeugung des Kindes zentral ist. Sind die Eltern überfürsorglich, sodass das Kind nicht die Erfahrung machen kann, dass ihm etwas zugetraut wird, kann die kindzentrierte Therapie wirksamer sein. Das Kind sollte die Erfahrung machen, dass ihm zugetraut wird, seine Ängste alleine bewältigen zu können. Auch hier zeigen sich reziproke Effekte: Das Selbstvertrauen der Kinder wird weiter gesteigert, wenn die Eltern realisieren, dass das Kind selbst gegen seine Ängste angehen kann, und sie ihm dadurch mehr zutrauen und mehr Autonomie gewähren [Kendall et al., 2003].

In der Depressionsbehandlung liegen keine systematischen Studien zur Effektivität einer zusätzlichen Elternarbeit vor. Bei Betroffenen im Jugendalter wird geraten, den Anteil der Elternarbeit in der Therapie möglichst gering zu halten, da Eltern zunehmend weniger bedeutsame Rollenvorbilder sind und eine von den Eltern unabhängige Therapiebeziehung wichtig sein kann, um den Ablösungsprozess von den Eltern zu unterstützen und den Selbstwert des Jugendlichen zu erhöhen [Pössel, 2009]. Bei der Auswahl des Settings in der Be- 
handlung der Depression spielen die Schwere, der bisherige Verlauf, die Motivation, mögliche Suizidalität sowie soziale und familiäre Ressourcen eine Rolle. Je nachdem muss aufgrund dieser Faktoren zwischen ambulanter und stationärer Behandlung entschieden werden [Ihle et al., 2004].

\section{Klassifikation und Diagnostik}

Die bevorstehende Neuauflage des Diagnostic and Statistic Manual of Mental Disorders (DSM, www.dsm5.org) hat im Bereich der Klassifikation einige Artikel hervorgebracht. So wird für das DSM-V eine Trennung zwischen Symptomen und klinischer Beeinträchtigung aufgrund der Symptomatik diskutiert. Die Beeinträchtigung und Belastung aufgrund einer Störung soll als Folge betrachtet werden und nicht als erforderliches Kriterium für die Vergabe einer Diagnose, sodass empfohlen wird, das Kriterium der klinischen Beeinträchtigung/Belastung aus den Diagnosekriterien zu entfernen [Rapee et al., 2012]. Rapee et al. [2012] fassen die Probleme des Kriteriums Beeinträchtigung wie folgt zusammen: Bei komorbiden Störungen sei es schwierig, die Beeinträchtigung einer spezifischen Störung zuschreiben zu können. Zudem sei das Konzept der klinischen Beeinträchtigung und des Leidensdrucks vage, subjektiv und tautologisch. Des Weiteren sei das Konzept inkonsistent mit demjenigen des International Classification of Diseases 10 (ICD-10). Bei Kindern kann als Einschränkung zusätzlich festgehalten werden, dass viele Kinder in diagnostischen Interviews mit der Frage nach der Beeinträchtigung Verständnisschwierigkeiten haben. Im Gegenzug kann angefügt werden, dass das Konzept der klinischen Beeinträchtigung klarer beschrieben werden könnte, z.B. durch Verhaltensbeschreibungen und Ankerwerte. Ein möglicher Nachteil eines Ausschlusses des Beeinträchtigungskriteriums ist eine Steigerung der Prävalenzraten [z.B. Merikangas et al., 2010]. Eine Empfehlung könnte sein, den «Schweregrad» der Symptome zu erhöhen. Zukünftig wird daher die objektive Erfassung vom Konstrukt Beeinträchtigung eine wichtige Rolle spielen [Rapee et al., 2012]. Ein kindgerechtes Verfahren zur Erfassung der Beeinträchtigung und Belastung («Beeinträchtigungs- und Belastungsrating») wird von In-Albon und Schneider [2012b] vorgestellt. Auf störungsspezifischer Ebene werden für das DSM-V folgende Änderungen diskutiert: Bei der Sozialen Phobie wird empfohlen, zukünftig den Begriff der Sozialen Angststörung zu verwenden, da die Betroffenen selten durchgängig soziale Situationen vermeiden, sondern diese häufig unter starker Angst ertragen. Des Weiteren soll der Subtypus «generalisiert» aufgegeben und ein neuer Subtypus, «nur Leistungsangst», aufgenommen werden [Bögels et al., 2010]. Ob die Prüfungsangst und das Nichtsprechen beim Selektiven Mutismus spezifische Subtypen der Sozialen Angststörung werden, muss in zukünftigen Studien untersucht werden. Bei der Störung mit Trennungsangst wird diskutiert, ob der Beginn vor dem 18. Le- bensjahr aufgehoben wird und ob die Anzahl der Symptome und die Dauer empirisch nachgewiesene Kriterien sind (www. dsm5.org). Mögliche Änderungen im DSM tragen vermutlich auch zu Veränderungen und stärkerer empirischer Evidenz der ICD-11-Kriterien bei. Eine Überarbeitung sollte beispielsweise darin bestehen, dass die Generalisierte Angststörung den anderen Angststörungen im Kindes- und Jugendalter nicht mehr hierarchisch übergeordnet wird (z.B. bei der Emotionalen Störung mit Trennungsangst, der Störung mit Sozialer Ängstlichkeit des Kindesalters, der Phobischen Störung des Kindesalters). Des Weiteren sollte das Kriterium der Einsicht, dass die Angst übertrieben und unbegründet ist, bei Kindern mit Angststörungen überprüft werden. Diese aufgeführten Punkte sind häufige Gründe für Diskrepanzen zwischen DSM und ICD, d.h. weshalb ein Kind die Kriterien einer ICD-Diagnose nicht erfüllt, während die DSM-Kriterien erfüllt sind. Für DSM-V wird auch wieder diskutiert, die «Störung mit Angst und Depression, gemischt» aufzunehmen. Die Diagnose war bereits in den Forschungskriterien des DSM-IV und ist im ICD-10 enthalten. Empirische Hintergründe sind die hohe Komorbiditätsrate von Angststörungen und Depression [Axelson und Birmaher, 2001] sowie gemeinsame ätiologische Faktoren [Barlow et al., 2004].

Mit der Klassifikation verbunden ist die Diagnostik. Auch in diesem Bereich findet eine stetige Weiterentwicklung statt. Eine reliable und valide Diagnosestellung ist eine zentrale Voraussetzung für eine erfolgreiche Therapie. Sowohl die Leitlinien zur Diagnostik und Behandlung von depressiven Störungen [Ihle et al., 2004] als auch die Leitlinien zur Diagnostik und Psychotherapie von Angst- und Phobischen Störungen im Kindes- und Jugendalter [Schneider und Döpfner, 2004] betonen den Einsatz von verschiedenen diagnostischen Methoden wie diagnostischen Interviews sowie Selbst- und Fremdbeurteilungsfragebögen. Eine aktuelle Übersicht liefern Barkmann et al. [2011] mit dem Buch «Klinisch-psychiatrische Ratingskalen für das Kindes- und Jugendalter». Weitere Materialien für die Diagnostik und Therapie sind im 4. Band des Lehrbuchs der Verhaltenstherapie [Meinlschmidt et al., 2012] zu finden.

\section{Prävention}

Für die Prävention, die Früherkennung und einen erleichterten Zugang zu Versorgungssystemen ist die Wissensvermittlung ein zentraler Schritt; zum einen, um Wissenslücken zu reduzieren, zum anderen, um Stigmatisierungen entgegenzuwirken. Im deutschsprachigen Raum liegen 2 evaluierte Broschüren vor, die zum Ziel haben, niederschwellig über Angst [Schneider und Borer, 2007] bzw. Depression bei Jugendlichen [Schiller und Allgaier, 2011] aufzuklären. Bei beiden Broschüren zeigten sich ein Wissenszuwachs und eine gute Akzeptanz [Schneider und Borer, 2003] sowie Einstellungsveränderungen [Allgaier et al., 2011]. Im deutschsprachigen 
Tab. 2. Empirisch validierte Interventionen und deutschsprachige Manuale für die einzelnen Angststörungen des Kindes- und Jugendalters [nach In-Albon, 2011]

\begin{tabular}{|c|c|c|}
\hline Angststörung & Empirisch validierte Interventionen & Therapiemanuale \\
\hline Verschiedene Angststörungen & $\begin{array}{l}\text { Psychoedukation, Abbau dysfunktionaler Gedanken, } \\
\text { graduierte Reizkonfrontation, operante Verfahren, } \\
\text { Entspannungsverfahren }\end{array}$ & $\begin{array}{l}\text { Coping Cat [Kendall und Hedtke, 2006] } \\
\text { FRIENDS [Barrett et al., 2000; deutsche } \\
\text { Übersetzung von Barrett et al., 2003] } \\
\text { Cool Kids [Lyneham et al., 2003] }\end{array}$ \\
\hline Spezifische Phobie & $\begin{array}{l}\text { 1-Session Behandlung; teilnehmendes Modell-Lernen; } \\
\text { kognitiv-verhaltenstherapeutische Interventionsprogramme mit } \\
\text { und ohne Elterntraining (einzeln und in der Gruppe): } \\
\text { Psychoedukation, Abbau dysfunktionaler Gedanken, graduierte } \\
\text { Reizkonfrontation, operante Verfahren, Entspannungsverfahren }\end{array}$ & $\begin{array}{l}\text { Derzeit kein störungsspezifisches Manual } \\
\text { verfügbar }\end{array}$ \\
\hline Soziale Phobie & $\begin{array}{l}\text { kognitiv-verhaltenstherapeutische Interventionsprogramme } \\
\text { mit und ohne Elterntraining (individuell oder in der Gruppe): } \\
\text { Psychoedukation, Abbau dysfunktionaler Gedanken, } \\
\text { soziales Kompetenz-/Selbstsicherheitstraining, graduierte } \\
\text { Reizkonfrontation }\end{array}$ & $\begin{array}{l}\text { Petermann und Petermann [2010] } \\
\text { Joormann und Unnewehr [2002] } \\
\text { Beck et al. [2006] } \\
\text { Tuschen-Caffier et al. [2009] } \\
\text { Büch und Döpfner [2012] }\end{array}$ \\
\hline Störung mit Trennungsangst & $\begin{array}{l}\text { kognitiv-verhaltenstherapeutische Interventionsprogramme mit } \\
\text { und ohne Elterntraining: Psychoedukation, Abbau dysfunktionaler } \\
\text { Gedanken, graduierte Reizkonfrontation, operante Verfahren }\end{array}$ & $\begin{array}{l}\text { Trennungsangstprogramm für Familien } \\
\text { (TAFF, Schneider, 2004, in Vorbereitung) }\end{array}$ \\
\hline Generalisierte Angststörung & $\begin{array}{l}\text { kognitiv-verhaltenstherapeutische Interventionsprogramme mit } \\
\text { und ohne Elterntraining: Psychoedukation, Abbau dysfunktionaler } \\
\text { Gedanken, graduierte Reizkonfrontation, Konfrontation in sensu, } \\
\text { operante Verfahren, Entspannungsverfahren }\end{array}$ & $\begin{array}{l}\text { Derzeit kein störungsspezifisches Manual } \\
\text { verfügbar }\end{array}$ \\
\hline Leistungsängste & $\begin{array}{l}\text { kognitiv-verhaltenstherapeutische Interventionsprogramme mit } \\
\text { und ohne Elterntraining: Psychoedukation, Abbau dysfunktionaler } \\
\text { Gedanken, graduierte Reizkonfrontation, operante Verfahren, } \\
\text { Entspannungsverfahren, Fertigkeitentraining }\end{array}$ & $\begin{array}{l}\text { Therapieprogramm für Kinder und } \\
\text { Jugendliche mit Angst- und } \\
\text { Zwangsstörungen (THAZ), Bd 1: } \\
\text { Leistungsängste [Suhr und Döpfner, 2005] }\end{array}$ \\
\hline
\end{tabular}

Raum liegen zudem empirisch abgesicherte Präventionsprogramme für Angst- und depressive Störungen vor. Das Training «Lars \& Lisa» [Pössel et al., 2003, 2004] wurde als universales Trainingsprogramm zur Prävention von Depression entwickelt. Zielgruppe sind Jugendliche im Alter von 14 und 15 Jahren. Methoden des Programms sind Selbstmanagement-Therapie, kognitive Umstrukturierung sowie soziales Kompetenz- und Selbstsicherheitstraining. Das Programm «FREUNDE» [Barrett et al., 2000, 2003] dient der universellen Prävention von Angst und Depression bei Kindern zwischen 7 und 12 Jahren. FREUNDE ist verhaltenstherapeutisch aufgebaut und hat zum Ziel, die Fähigkeiten und Techniken der 3 Komponenten physiologischer Bereich, kognitiver Bereich und Lernen zu vermitteln. Verschiedene Studien haben die Wirksamkeit und Akzeptanz des Programms untersucht und belegt [Barrett et al., 2003, 2006; Essau et al., 2004]. Eimecke et al. [2010] ergänzten das FREUNDE-Training um ein Elterntraining und untersuchten dessen Wirksamkeit in der indizierten Prävention bei 8- bis 12-jährigen Kindern. Das Elterntraining hatte keinen zusätzlichen Effekt auf die Veränderung introversiver Symptome. Jedoch erlebten sich alle Eltern nach dem Training kompetenter im Umgang mit ihren Kindern. «GO!» [Junge et al., 2002, 2007] ist als universelles Trainingsprogramm für Jugendliche zwischen 14 und 18 Jahren zur Prävention von Angst und Depression konzipiert. Das Konzept basiert auf kognitiv-verhaltenstherapeutischen Modellen, wobei sich die Elemente auf Angst, Depression, Training sozialer Kompetenzen und Stressbewältigung beziehen.

Die Setting-Frage stellt sich auch in der Präventionsforschung. Eine kürzlich erschienene Studie verweist darauf, dass, wie in der Psychotherapie für Angststörungen auch, in der Prävention kein Wirksamkeitsunterschied zwischen kind- und elternfokussiertem Programm besteht [Simon et al., 2011]: Sowohl in der eltern- als auch kindfokussierten Gruppe zeigte sich eine Angstreduktion. In der universellen Prävention stellt sich die Frage, inwieweit Programme störungsspezifisch sein müssen oder ob es nicht sogar sinnvoller wäre, Programme durchzuführen, die die psychische Gesundheit allgemein fördern. Gemeint sind dabei z.B. Programme zur Verbesserung der Emotionsregulation und zur Steigerung der sozial-emotionalen Kompetenzen und des Selbstwerts. Petermann et al. [2007] entwickelten für Kinder im Grundschulalter ein Training zur Förderung emotionaler, sozialer und moralischer Entwicklung. Die Kinder lernen beispielsweise, Gefühle zu erkennen und angemessen mit Gefühlen und Konfliktsituationen umzugehen. Die Ergebnisse zeigten eine Zunahme sozialer Kompetenzen und eine Abnahme sozial-emotionaler Probleme [von Marées und Petermann, 2009]. 


\section{Therapieinhalte}

In den vergangenen Jahren sind einige empirisch validierte Therapiemanuale für die Behandlung von Angststörungen erschienen. Wie in Tabelle 2 ersichtlich, sind jedoch störungsspezifische Angstmanuale bislang eher selten. Die am besten überprüften Programme wurden für verschiedene Angststörungen entwickelt. Dabei zeigen sich über die Manuale hinweg geringe Veränderungen in den Inhalten. Nachdem immer mehr Komponenten in die Therapien aufgenommen wurden, zeigte sich jedoch, dass weniger oft mehr ist und dass es nicht sinnvoll ist, die Therapiesitzungen mit möglichst vielen Komponenten zu ergänzen. Es sollte genügend Zeit zur Verfügung stehen, das Gelernte umzusetzen und zu üben. Zudem sollten bezüglich der Konfrontationsübungen neue Forschungserkenntnisse aufgenommen werden, z.B. dass es für eine erfolgreiche Konfrontationstherapie entscheidend ist, diese in verschiedenen Kontexten durchzuführen. Zudem scheint die Verfügbarkeit von Hinweisreizen für Patienten hilfreich zu sein, da sie diese außerhalb des Therapiekontextes in neue Situationen mitnehmen können [Arch und Craske, 2009]. In der Therapie mit Kindern können dies beispielsweise ein Mutmach-Stein oder ein Mutmach-Kärtchen sein, die sich leicht in der Hosentasche und Schultasche unterbringen lassen.

Für die Behandlung depressiver Störungen liegen, wie im Abschnitt zur Psychotherapieforschung beschrieben, 2 Manuale vor, für die aber RCTs noch ausstehen [Ihle und Herrle, 2003; Harrington, 2001].

Gemeinsame Bausteine in der Therapie von Angst- und depressiven Störungen sind [Ihle et al., 2004; In-Albon, 2011; Pössel, 2009]: 1) Psychoedukation, 2) kognitive Interventionen (Selbstinstruktions- und Problemlösetrainings), 3) verhaltensorientierte Techniken (Angststörungen: Abbau Vermeidungsverhalten durch Konfrontationsübungen; depressive Störungen: Aktivitätsaufbau) und 4) Rückfallprophylaxe. Folgende zusätzliche Komponenten können in den Therapien sinnvoll sein: Entspannungsverfahren, Förderung der emotionalen und sozialen Kompetenzen.

Aufgrund der hohen Komorbidität von Angststörungen und Depressionen entwickelten Barlow et al. [2011] ein transdiagnostisches Therapieprogramm zur Behandlung von Angst- und depressiven Störungen für Erwachsene. Theoretischer Hintergrund ist das Tripartite-Modell, das zeigte, dass negative Affekte und geringe positive Affekte übergeordnete Faktoren der unipolaren Depression und verschiedener Angststörungen sind [Chorpita et al., 1998]. Dies konnte auch für das Kindes- und Jugendalter bestätigt werden [Chorpita, 2002]. Das Programm ist modulartig aufgebaut und beinhaltet 3 Hauptstrategien: 1) Änderung und Neubewertung kognitiver Prozesse, 2) Prävention emotionaler Vermeidung und 3) Verhaltensänderung [Allen et al., 2005]. Das Programm wurde für das Kindes- und Jugendalter angepasst, und Evaluationsstudien zur Gruppentherapie werden derzeit durchgeführt [Ehrenreich et al., 2009; Ehrenreich et al., 2012].

\section{Zusammenfassung und Ausblick}

Ist nun die Verhaltenstherapie bei Kindern mit Angst- und depressiven Störungen aus den Kinderschuhen ausgewachsen? Diese Frage aus der Einleitung kann zum heutigen Zeitpunkt weder eindeutig verneint noch bejaht werden. Seit dem Erscheinen des Sonderhefts zur Verhaltenstherapie bei Kindern und Jugendlichen im Jahre 2000 sind viele Studien erschienen, die aufzeigen, dass internalisierende Störungen im Kindesund Jugendalter häufig und stabil sind sowie einen beträchtlichen Risikofaktor für die Entwicklung psychischer Störungen im Erwachsenenalter darstellen. Dennoch werden diese «stillen Störungen» häufig übersehen und betroffene Kinder und Jugendliche erhalten eine unzureichende Versorgung. Diese aufgeführten Fakten sprechen jedoch klar für eine frühe Erkennung, Prävention und wirksame Behandlung internalisierender Störungen. Risikoreiche Entwicklungsverläufe von Kindern und Jugendlichen können mithilfe von Präventionsund Interventionsprogrammen positiver gestaltet werden. Voraussetzung für die Entwicklung wirksamer Präventions- und Behandlungsprogramme ist eine gute Kenntnis der Risikound Schutzfaktoren. Auf der Basis des derzeitigen Forschungsstands fehlt jedoch ein umfassendes, empirisch fundiertes Modell zur Wirkung von Schutz- und Risikofaktoren.

Die Anzahl publizierter kontrollierter Psychotherapiestudien hat in den vergangenen 12 Jahren beträchtlich zugenommen. Zusammengefasst kann festgehalten werden, dass insbesondere die Verhaltenstherapie bei Angststörungen im Kindes- und Jugendalter und bei depressiven Störungen im Jugendalter nachhaltig wirksam ist. Falls eine pharmakologische Behandlung erforderlich ist, sind SSRIs die Behandlung erster Wahl. Klinisch relevante Erkenntnisse liegen aus dem Bereich der Setting-Frage vor. Der derzeitige Stand der Forschung zeigt, dass keine Wirksamkeitsunterschiede vorliegen, wenn die Verhaltenstherapie individuell oder in der Gruppe, kindfokussiert oder mit Einbezug der Eltern durchgeführt wird. Für welches Kind bzw. welche Familie welches Setting optimal ist, fehlen aber noch Hinweise. Weitere Forschung ist zudem notwendig im Bereich der effectiveness-Wirksamkeit, der Behandlung von Kindern im Vorschulalter bzw. von Jugendlichen, der störungsspezifischen Therapiestudien und der Wirksamkeit einzelner Therapiekomponenten. Im Hinblick auf die Überarbeitung der Klassifikationssysteme muss abgewartet werden, wie sich die Veränderungen auf die Vergabe von Diagnosen und Prävalenzraten von psychischen Störungen im Kindes- und Jugendalter auswirken werden. Mögliche Änderungen in den Diagnosekriterien führen wiederum zu Anpassungen in diagnostischen Verfahren, z.B. klinisch diagnostischen Interviews, die an die Klassifikationssysteme angelehnt sind. Da die reliable und valide Diagnostik eine zentrale Voraussetzung für eine erfolgreiche Therapie ist, findet in diesem Bereich eine stetige Weiterentwicklung statt. Für die Zukunft bleibt zu klären, wie internalisierenden Störungen präventiv vorgebeugt werden kann. Eine Möglichkeit könnte 
die Wissensverbreitung zur Erkennung von Angst- und Depressionssymptomen durch Pädiater und Lehrpersonen sein, aber auch durch die Schulung von Jugendlichen selbst, z.B. mit Aufklärungsbroschüren. Ein Faktor, der einen Prädiktor und nicht nur ein Epiphänomen für internalisierende Störungen darstellt, ist der Selbstwert [Sowislo und Orth, 2012]. Daher sollte der Selbstwert in Präventionsprogrammen für die psychische Gesundheit eine zentrale Rolle einnehmen. Für die Therapie von Angststörungen liegen empirisch überprüfte deutschsprachige Manuale vor. Die bestehenden deutschsprachigen Therapiemanuale für depressive Störungen müssen noch auf ihre Wirksamkeit überprüft werden. Neben der Steigerung der Wirksamkeit von vorhandenen Programmen ist die Verbreitung der empirisch validierten Programme in die klinische Praxis eine zentrale Aufgabe.

\section{Disclosure Statement}

Hiermit bestätige ich, dass keine Interessenskonflikte vorliegen.

\section{Referenzen}

AACAP: Practice parameter for the assessment and

treatment of children and adolescents with anxiety disorder. J Am Acad Child Adolesc Psychiatry 2007:46:267-283.

Allen LB, Ehrenreich JT, Barlow DH: A unified treatment for emotional disorders: applications with adults and adolescents. Jpn J Behav Ther 2005;31: 3-31.

Allgaier A-K, Schiller Y, Schulte-Körne G: Wissens-

$\checkmark$ und Einstellungsänderungen zu Depression im Jugendalter - Entwicklung und Evaluation einer Aufklärungsbroschüre. Kindheit und Entwicklung 2011;20:247-255.

Arch JJ, Craske MG: First-line treatment: a critical ap-

$\checkmark$ praisal of cognitive behavioral therapy developments and alternatives. Psychiatr Clin North Am 2009:32:525-547.

Axelson DA, Birmaher B: Relation between anxiety

and depressive disorders in childhood and adolescence. Depress Anxiety 2001;14:67-78.

Bachmann M, Bachmann CJ, John K, Heinzel-Guten-

brunner M, Remschmidt H, Mattejat F: The effectiveness of child and adolescent psychiatric treatments in a naturalistic outpatient setting. World Psychiatry 2010;9:111-117.

Barlow DH, Allen LB, Choate ML: Toward a unified

treatment for emotional disorders. Behav Ther 2004;35:205-230.

Barlow DH, Farchione TJ, Fairholme CP, Ellard KK Boisseau CL, Allen LB, Ehrenreich-May J: Unified Protocol for Transdiagnostic Treatment of Emotional Disorders: Therapist Guide. New York, Oxford University Press, 2011.

Barrett P, Webster H, Turner C: FRIENDS for Children. Bowen Hills, Australian Academic Press,

Barrett P, Webster H, Turner C, Essau CE, Conradt J: FREUNDE für Kinder. München, Reinhardt, 2003.

Barrett PM, Farrell LJ, Ollendick TH, Dadds M

Long-term outcomes of an Australian universal prevention trial of anxiety and depression symptoms in children and youth: an evaluation of the FRIENDS program. J Clin Child Adolesc Psycho 2006;35:403-411.

Barkmann C, Schulte-Markwort M, Brähler E: Klinisch-psychiatrische Ratingskalen für das Kindesund Jugendalter. Göttingen, Hogrefe, 2011.

Beck N, Cäsar S, Leonhardt B: Training sozialer Fähigkeiten. Tübingen, DGVT, 2006.

Birmaher B, Ryan ND, Williamson DE, Brent DA,

Kaufman J, Dahl RE, Perel J, Nelson B: Childhood and adolescent depression: a review of the past 10 years. Part I. J Am Acad Child Adolesc Psychiatry 1996;35:1427-1439.
Bodden DH, Bögels SM, Nauta MH, De Haan E, Ringrose J, Appelboom C, Brinkman AG, Appelboom-Geerts KC: Child versus family cognitivebehavioral therapy in clinically anxious youth: an efficacy and partial effectiveness study. J Am Acad Child Adolesc Psychiatry 2008;47:1384-1394.

Bögels SM, Alden L, Beidel DC, Clark LA, Pine DS,

Stein MB, Voncken M: Review - social anxiety disorder: questions and answers for the DSM-V. Depress Anxiety 2010;27:168-189.

Brent DA: Glad for what TADS adds, but many

TADS grads still sad. J Am Acad Child Adolesc Psychiatry 2006;45:1461-1464.

Brent DA, Holder D, Kolko D, Birmaher B, Baugher

M, Roth C, Iyengar S, Johnson BA: A clinical psychotherapy trial for adolescent depression comparing cognitive, family, and supportive treatments. Arch Gen Psychiatry 1997;54:877-885.

Brent D, Emslie G, Clarke G, Wagner KD, Asarnow

JR, Keller M, Vitiello B, Ritz L, Iyengar S, Abebe K, Birmaher B, Ryan N, Kennard B, Hughes C, DeBar L, McCracken J, Strober M, Suddath R, Spirito A, Leonard H, Melhem N, Porta G, Onorato $\mathrm{M}$, Zelazny J: Switching to another SSRI or to venlafaxine with or without cognitive behavioral therapy for adolescents with SSRI-resistant depression: the TORDIA randomized controlled trial. JAMA 2008;299:901-913.

Bridge JA, Iyengar S, Salary CB, Barbe RP, Birmaher B, Pincus HA, Ren L, Brent DA: Clinical response and risk for reported suicidal ideation and suicidal attempts in pediatric antidepressant treatment. JAMA 2007;297:1683-1696.

Brückl TM, Wittchen HU, Höfler M, Pfister H, Schneider S, Lieb R: Childhood separation anxiety and the risk for subsequent psychopathology: results from a community study. Psychother Psychosom 2007;76:47-56.

Büch H, Döpfner M: Soziale Ängste. Therapieprogramm für Kinder und Jugendliche mit Angst und Zwangsstörungen (THAZ), Bd 2. Göttingen, Hogrefe, 2012.

Carter AS, Wagmiller RJ, Gray SAO, McCarthy KJ, Horwitz SM, Briggs-Gowan MJ: Prevalence of DSM-IV disorder in a representative, healthy birth cohort at school entry: sociodemographic risks and social adaptation. J Am Acad Child Adolesc Psychiatry 2010;49:686-698

Casey RJ, Berman JS: The outcome of psychotherapy with children. Psychol Bull 1985;98:388-400.

Chorpita BF: The tripartite model and dimensions of

anxiety and depression: an examination of structure in a large school sample. J Abnorm Child Psychol 2002;30:177-190.
Chorpita BE, Albano AM, Barlow DH: The structure of negative emotions in a clinical sample of children and adolescents. J Abnorm Child Psychol 1998;107: $74-85$.

Copeland WE, Shanahan L, Costello EJ, Angold A

Childhood and adolescent psychiatric disorders as predictors of young adult disorders. Arch Gen Psychiatry 2009;66:764-772.

Costello EJ, Erkanli A, Angold A: Is there an epi-

demic of child or adolescent depression? J Child Psychol Psychiatry 2006;47:1263-1271.

Cowart MJ, Ollendick TH: Attention training in so-

cially anxious children: a multiple baseline design analysis. J Anxiety Disord 2011;25:972-977.

Diamond GS, Reis BF, Diamond GM, Siqueland L,

Isaacs L: Attachment-based family therapy for depressed adolescents: a treatment development study. J Am Acad Child Adolesc Psychiatry 2002; 41:1190-1196

Eimecke S, Pauschardt J, Mattejat F: Prevention of childhood anxiety and depression: efficacy of an additional parent training program. Verhaltenstherapie 2010;20:193-200

Ehrenreich JT, Goldstein CM, Wright LR, Barlow

DH: Development of a unified protocol for the treatment of emotional disorders in youth. Child Fam Behav Ther 2009;31:20-37.

Ehrenreich May J, Laird EA, Guttman LE: The devel-

opment of a transdiagnostic, cognitive behavioral group intervention for childhood anxiety disorders and co-occurring depression symptoms. Cogn Behav Pract 2012;19:41-55

Emmelkamp PMG, Wittchen HU: Specific phobias; in Andrews G, Charney DS, Sirovatka PJ, et al. (eds) Stress-Induced and Fear Circuitry Disorders. Refining the Research Agenda for DSM-IV. Arlington, APA, 2009.

Essau CA: Frequency and patterns of mental health

services utilization among adolescents with anxiety and depressive disorders. J Depress Anxiety 2005; 22:130-137.

Essau CA, Conradt J, Reiss AL: Klassifikation, diagnostisches Vorgehen und Epidemiologie; in Schneider S (ed): Angststörungen bei Kindern und Jugendlichen. Berlin, Springer, 2004, pp 79-102.

Harrington RC: Kognitive Verhaltenstherapie bei depressiven Kindern und Jugendlichen. Göttingen, Hogrefe, 2001.

Hirshfeld-Becker DR, Masek B, Henin A, Blakely LR,

Pollock-Wurman RA, McQuade J, DePetrillo L, Briesch J, Ollendick TH, Rosenbaum JF, Biederman $\mathrm{J}$ : Cognitive behavioral therapy for 4- to 7-year-old children with anxiety disorders: a randomized clinical trial. J Consult Clin Psychol 2010;78:498-510. 
Hudson J, Rapee R: Psychopathology and the Family. Oxford, Elsevier, 2005.

Ihle W, Esser G: Epidemiologie psychischer Störun-

gen im Kindes- und Jugendalter: Prävalenz, Verlauf, Komorbidität und Geschlechtsunterschiede. Psychol Rundsch 2002;53:159-169.

Ihle W, Herrle J: Prävention, Behandlung und Rückfallprophylaxe depressiver Störungen im Jugendalter. Tübingen, DGVT, 2003.

Ihle W, Ahle MA, Jahnke D, Esser G: Leitlinien zur

Diagnostik und Psychotherapie von depressiven Störungen im Kindes- und Jugendalter: Ein evidenzbasierter Diskussionsvorschlag. Kindheit und Entwicklung 2004;13:64-79.

In-Albon T: Kinder und Jugendliche mit Angststörungen. Stuttgart, Kohlhammer, 2011.

In-Albon T, Schneider S: Psychotherapy of childhood

anxiety disorders: a meta-analysis. Psychother Psychosom 2007;76:15-24.

In-Albon T, Zumsteg U, Müller D, Schneider S: Psychological problems in the pediatric setting - results of a Swiss survey. Swiss Med Wkly 2010;140:w13092.

In-Albon T, Schneider S: Does the vigilance-avoid-

ance gazing behaviour of children with separation anxiety disorder change after cognitive-behavioral therapy? J Abnorm Child Psychol 2012a;40:11491156.

In-Albon T, Schneider S: Verlaufsdiagnostik und Therapieevaluation bei Kindern und Jugendlichen in Meinlschmidt G, Schneider S, Margraf J (eds): Materialien für die Psychotherapie. Lehrbuch der Verhaltenstherapie, Bd 4. Berlin, Springer, 2012b, pp 515-522.

James A, Soler A, Weatherall R: Cognitive behavioural therapy for anxiety disorders in children and adolescents. Cochrane Database Syst Rev 2005: CD004690.

Joormann J, Unnewehr S: Behandlung der Sozialen Phobie bei Kindern und Jugendlichen. Göttingen, Hogrefe, 2002.

Junge J, Neumer S, Manz R, Margraf J: Angst und Depression im Jugendalter vorbeugen. GO! - Ein Programm für Gesundheit und Optimismus. Weinheim, PVU Beltz, 2002.

Junge J, Annen B, Margraf J: GO! - Ein Programm zur Prävention von Angst und Depression be Jugendlichen. Langzeiteffekte und Weiterentwicklungen; in Röhrle B (ed): Prävention und Gesundheitsförderung für Kinder und Jugendliche. Tübingen, DGVT, 2007.

Khanna MS, Kendall PC: Computer-assisted cognitive

behavioural therapy for child anxiety. J Consult Clin Psychol 2010;78:737-745.

Kendall PC: Treating anxiety disorders in children

results of a randomized clinical trial. J Consult Clin Psychol 1994;62:100-110.

Kendall PC, Flannery-Schroeder E, Panichelli-Minde

SM, Southam-Gerow M, Henin A, Warman M Therapy for youths with anxiety disorders: a second randomized clinical trial. J Consult Clin Psychol 1997;65:366-380

Kendall PC, Aschenbrand SG, Hudson JL: Child-focused treatment of anxiety; in Kazdin AE, Weisz JR (eds): Evidence-Based Psychotherapies for Children and Adolescents. New York, Guilford Press, 2003, pp 81-100

Kendall PC, Safford S, Flannery-Schröder E, Webb A

Child anxiety treatment: outcomes in adolescence and impact on substance use and depression at 7.4-year follow-up. J Consult Clin Psychol 2004;72 276-287.

Kendall PC, Hedtke K: Coping Cat Workbook, ed 2. Ardmore, Workbook Publishing, 2006.
Kessler RC, Berglund P, Demler O, Jin R, Merikangas

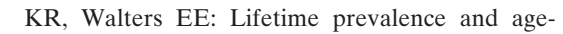
of-onset distributions of DSM-IV disorders in the National Comorbidity Survey Replication. Arch Gen Psychiatry 2005;62:593-602.

Kim-Cohen J, Caspi A, Moffitt TE, Harrington H,

Milne BJ, Poulton R: Prior juvenile diagnoses in adults with mental disorder: developmental followback of a prospective-longitudinal cohort. Arch Gen Psychiatry 2003;60:709-717.

Klein JB, Jacobs RH, Reinecke MA: Cognitive-behav-

ioral therapy for adolescent depression: a metaanalytic investigation of changes in effect-size estimates. J Am Acad Child Adolesc Psychiatry 2007; 46:1403-1413.

Kremberg E, Mitte K: Kognitiv-behaviorale und beha-

viorale Interventionen der sozialen Phobie im Kindes- und Jugendalter. Z Klin Psychol Psychother 2005:34:196-204.

Kuschel A, Heinrichs N, Bertram H, Naumann S,

Hahlweg K: Psychische Auffälligkeiten bei Kindergartenkindern aus der Sicht der Eltern und Erzieherinnen in Abhängigkeit von soziodemographischen Merkmalen. Kindheit und Entwicklung 2008; 17:161-172.

Lewinsohn P, Clarke GN: Psychosocial treatments for adolescent depression. Clin Psychol Rev 1999;19: 329-342.

Lyneham HJ, Abbott MJ, Wignall A, Rapee RM: The Cool Kids Family Program - Therapist Manual. Sydney, Macquarie University, 2003.

Meinlschmidt G, Schneider S, Margraf J: Materialien für die Psychotherapie. Lehrbuch der Verhaltenstherapie, Bd 4. Berlin, Springer, 2012.

Meltzer H, Gatward R, Goodman R, Ford T: Mental health of children and adolescents in Great Britain. London, Stationery Office, 2000.

Melfsen S, Kühnemund M, Schwieger J, Warnke A, Stadler C, Poustka F, Stangier U: Cognitive behavioral therapy of socially phobic children focusing on cognition: a randomised wait-list control study. Child Adolesc Psychiatry Ment Health 2011;5:1-12. Merikangas KR, He J, Burstein M, Swanson SA, Avenevoli S, Cui L, Benjet C, Georgiades K, Swendsen $\mathrm{J}$ : Lifetime prevalence of mental disorders in U.S. adolescents - results from the National Comorbidity Survey Replication-Adolescent Supplement (NCS-A). J Am Acad Child Adolesc Psychiatry 2010;49:980-989.

Mufson L, Weissman MM, Moreau D, Garfinkel R:

Efficacy of interpersonal psychotherapy for depressed adolescents. Arch Gen Psychiatry 1999;56: 573-579.

NIMH (The National Advisory Mental Health Council Workgroup on Child and Adolescent Mental Health Intervention Development and Deployment): Blueprint for Change: Research in Child and Adolescent Mental Health. Washington, NIMH, 2001.

Ollendick TH, Öst L-G, Reuterskiöld L, Costa N,

Cederlund R, Sirbu C, Davis TE 3rd, Jarrett MA: One-session treatment of specific phobias in youth: a randomized clinical trial in the United States and Sweden. J Consult Clin Psychol 2009;77:504-51.

Petermann P: Zur Epidemiologie psychischer Störungen im Kindes- und Jugendalter. Eine Bestandsaufnahme. Kindheit und Entwicklung 2005;14:48-57.

Petermann F, Koglin U, Natzke H, von Marées N: Verhaltenstraining in der Grundschule. Ein Präventionsprogramm zur Förderung emotionaler und sozialer Kompetenzen. Göttingen, Hogrefe, 2007.

Petermann F, Petermann U: Training mit Jugendlichen, Förderung von Arbeits- und Sozialverhalten, ed 9. Göttingen, Hogrefe, 2010.
Pössel P: Depression/Suizidalität; in Schneider S, Margraf J (eds): Lehrbuch der Verhaltenstherapie, Bd 3. Berlin, Springer, 2009, pp 663-687.

Pössel P, Horn AB, Hautzinger M: Erste Ergebnisse eines Programms zur schulbasierten Prävention von depressiven Symptomen bei Jugendlichen. Z Gesund Psychol 2003;11:10-20.

Pössel P, Horn AB, Seemann S, Hautzinger M: Lust an realistischer Sicht und Leichtigkeit im sozialen Alltag - LARS \& LISA. Manual eines schulbasierten universalen Schulprogramms von Depression bei Jugendlichen. Göttingen, Hogrefe, 2004.

Pössel P, Hautzinger M: Effekte pharmakologischer

und psychotherapeutischer Interventionen auf Depressionen bei Kindern und Jugendlichen. Z Kinder Jugendpsychiatr Psychother 2006;34:243-255.

Rapee RM, Bögels SM, van der Sluis CM, Craske

MG, Ollendick T: Annual research review - conceptualising functional impairment in children and adolescents. J Child Psychol Psychiatry 2012;53. 454-468.

Reinecke MA, Ryan NE, DuBois DL: Cognitive-be-

havioral therapy of depression and depressive symptoms during adolescence: a review and metaanalysis. J Am Acad Child Adolesc Psychiatry 1998;37:26-34.

Rosselló J, Bernal G: The efficacy of cognitive-behav-

ioral and interpersonal treatments for depression in Puerto Rican adolescents. J Consult Clin Psychol 1999;67:734-745.

Rynn M, Puliafico A, Heleniak C, Rikhi P, Ghalib K, Vidair H: Advances in pharmacotherapy for pediatric anxiety disorders. Depress Anxiety 2011;28:76-87.

Saavedra LM, Silverman WK, Morgan-Lopez AA,

Kurtines WM: Cognitive behavioral treatment for childhood anxiety disorders: long-term effects on anxiety and secondary disorders in young adulthood. J Child Psychol Psychiatry 2010;51:924-934.

Schiller Y, Allgaier A-K: Paul ganz unten - Depressionen bei Jugendlichen verstehen. Aufklärungsbroschüre der Klinik und Poliklinik für Kinder- und Jugendpsychiatrie, Psychosomatik und Psychotherapie, Klinikum der Universität München, 2011.

Schneider S: Steckt die Verhaltenstherapie bei Kin-

dern noch in den Kinderschuhen? Verhaltenstherapie 2000;10:65-66.

Schneider S, Borer S: Primäre Prävention von Angst-

störungen: Evaluation einer Broschüre für Kinder und Jugendliche. Kindheit und Entwicklung 2003; 12:111-118.

Schneider S, Döpfner M: Leitlinien zur Diagnostik

$\checkmark$ und Psychotherapie von Angst- und Phobischen Störungen im Kindes- und Jugendalter: Ein evidenzbasierter Diskussionsvorschlag. Kindheit und Entwicklung 2004;13:80-96.

Schneider S, Borer S: Nur keine Panik. Was Kids über Angst wissen sollten, ed 2. Basel, Karger, 2007.

Schneider S, Houweling JEG, Gommlich-Schneider S,

Klein C, Nündel B, Wolke D: Effect of maternal panic disorder on mother-child interaction and relation to child anxiety and child self-efficacy. Arch Womens Ment Health 2009;12:251-259.

Schneider S, Blatter-Meunier J, Herren C, Adornetto C

In-Albon T, Lavallee K: Disorder-specific cognitivebehavioral therapy for separation anxiety disorder in young children: a randomized waiting-list-controlled trial. Psychother Psychosom 2011:80:206-215.

Silverman WK, Pina AA, Viswesvaran C: Evidence-

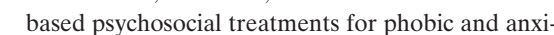
ety disorders in children and adolescents. J Clin Child Adolesc Psychol 2008;37:105-130.

Silverman WK, Kurtines WM, Jaccard J, Pina AA:

Directionality of change in youth anxiety treatment involving parents: an initial examination. J Consult Clin Psychol 2009;77:474-485. 
Siqueland L, Rynn M, Diamond GS: Cognitive behavioral and attachment based family therapy for anxious adolescents: phase I and phase II studies. J Anxiety Disord 2005;19:361-381.

Simon E, Bögels SM, Voncken JM: Efficacy of child-focused and parent-focused

interventions in a child anxiety prevention study. J Clin Child Adolesc Psychol 2011:40:204-219.

Sowislo JF, Orth U: Does low self-esteem predict depression and anxiety? A metaanalysis of longitudinal studies. Psychol Bull DOI: 10.1037/a0028931.

Spence SH, Donovan CL, March S, Gamble A, Anderson RE, Prosser S, Kenardy J:

A randomized controlled trial of online versus clinic-based CBT for adolescent anxiety. J Consult Clin Psychol 2011;79:629-642.

Steinhausen HC: Psychische Störungen bei Kindern und Jugendlichen, ed 7. München, Urban und Fischer, 2010.

Steinhausen HC, Winkler Metzke C, Meier M, Kannenberg R: Prevalence of child

and adolescent psychiatric disorders: the Zürich epidemiological study. Acta Psychiatr Scand 1998;98:261-271.

Suhr L, Döpfner M: Diagnostik und Therapie von Leistungsängsten. Therapieprogramm für Kinder und Jugendliche mit Angst- und Zwangsstörungen (THAZ), Bd 1. Göttingen, Hogrefe, 2005.

Treatment for Adolescents with Depression Study (TADS) Team: Fluoxetine, cog-

nitive-behavioral therapy, and their combination for adolescents with depression. JAMA 2004:292:807-820.

Treatment for Adolescents with Depression Study (TADS) Team: Treatment for

Adolescents with Depression Study: long-term effectiveness and safety outcomes. Arch Gen Psychiatry 2007;64:1132-1144.

Tuschen-Caffier B, Kühl S, Bender C: Soziale Ängste und soziale Angststörung im Kindes- und Jugendalter. Ein Therapiemanual. Göttingen, Hogrefe, 2009.

Tuschen-Caffier B, Krämer M, Seefeldt WL, Heinrichs N: Evaluation of a cognitivebehavioral group treatment for childhood social anxiety disorder in a randomized clinical sample. Symposium at the 6th World Congress of Behavioral and Cognitive Therapies. Boston, USA, 2010.

von Marées N, Petermann F: Förderung sozial-emotionaler Kompetenzen im

Grundschulalter. Kindheit und Entwicklung 2009;18:244-253.

Walkup JT, Albano AM, Piacentini J, Birmaher B, Compton SN, Sherrill JT, Gins-

burg GS, Rynn MA, McCracken J, Waslick B, Iyengar S, March JS, Kendall PC: Cognitive behavioral therapy, sertraline, or a combination in childhood anxiety. New Engl J Med 2008;359:2753-2766.

Weiss B, Catron T, Harris V, Phung TM: The effectiveness of traditional child psy-

chotherapy. J Consult Clin Psychol 1999;67:82-94.

Weiss B, Catron T, Harris V: A 2-year follow-up of the effectiveness of traditional

child psychotherapy. J Consult Clin Psychol 2000;68:1094-1101.

Weisz JR, McCarty CA, Valeri SM: Effects of psychotherapy for depression in chil-

dren and adolescents: a meta-analysis. Psychol Bull 2006;132:132-149.

Wittchen HU, Nelson CB, Lachner G: Prevalence of mental disorders and psycho-

social impairments in adolescents and young adults. Psychol Med 1998;28:109-126.

Wittchen H-U, Kessler RC, Pfister H, Lieb M: Why do people with anxiety disor-

ders become depressed? A prospective-longitudinal community study. Acta Psychiatr Scand 2000a;102:14-23.

Wittchen H-U, Lieb R, Pfister H, Schuster P: The waxing and waning of mental

disorders: evaluating the stability of syndromes of mental disorders in the population. Compr Psychiatry 2000b;41:122-132.

Woodward LJ, Fergusson DM: Life course outcomes of young people with anxiety

disorders in adolescence. J Am Acad Child Adolesc Psychiatry 2001;40:10861093. 